\title{
On the massive star content of the nearby dwarf irregular Wolf-Rayet galaxy IC $4662^{\star}$
}

\author{
P. A. Crowther and J. L. Bibby
}

\begin{abstract}
Department of Physics \& Astronomy, Hicks Building, University of Sheffield, Hounsfield Road, Sheffield S3 7RH, UK e-mail: Paul.Crowther@sheffield.ac.uk
\end{abstract}

Received 30 January 2009 / Accepted 11 March 2009

\section{ABSTRACT}

\begin{abstract}
Aims. We investigate the massive stellar content of the nearby dwarf irregular Wolf-Rayet galaxy IC 4662, and consider its global star forming properties in the context of other metal-poor galaxies, namely the SMC, IC 10 and NGC 1569.

Methods. Very Large Telescope/FORS2 imaging and spectroscopy plus archival Hubble Space Telescope/ACS imaging datasets permit us to spatially identify the location, number and probable subtypes of Wolf-Rayet stars within this galaxy. We also investigate suggestions that a significant fraction of the ionizing photons of the two giant $\mathrm{H}$ II regions A1 and A2 lie deeply embedded within these regions.

Results. Wolf-Rayet stars are associated with a number of sources within IC 4662-A1 and A2, plus a third compact H II region to the north west of A1 (A1-NW). Several sources appear to be isolated, single (or binary) luminous nitrogen sequence WR stars, while extended sources are clusters whose masses exceed the Orion Nebula Cluster by, at most, a factor of two. IC 4662 lacks optically visible young massive, compact clusters that are common in other nearby dwarf irregular galaxies. A comparison between radio and $\mathrm{H} \alpha$-derived ionizing fluxes of A1 and A2 suggests that 30-50\% of their total Lyman continuum fluxes lie deeply embedded within these regions.

Conclusions. The star formation surface density of IC 4662 is insufficient for this galaxy to qualify as a starburst galaxy, based upon its photometric radius, $R_{25}$. If instead, we were to adopt the $V$-band scale length $R_{\mathrm{D}}$ from Hunter \& Elmegreen, IC 4662 would comfortably qualify as a starburst galaxy, since its star formation intensity would exceed $0.1 M_{\odot} \mathrm{yr}^{-1} \mathrm{kpc}^{-2}$.
\end{abstract}

Key words. galaxies: individual: IC 4662 - stars: Wolf-Rayet - ISM: HII regions - galaxies: star clusters - galaxies: starburst galaxies: dwarf

\section{Introduction}

Star-forming galaxies at high redshift, such as Lyman break galaxies, possess metallicities comparable to the Small and Large Magellanic Clouds (SMC, LMC), although typically with star formation surface densities many orders of magnitude higher. Within the local universe, there are no direct analogues of massive, metal-deficient Lyman break galaxies, so one must rely on lower mass, dwarf irregular galaxies to study starburst activity at low metallicity.

Dwarf, metal-poor starburst galaxies such as NGC 1569 (Buckalew \& Kobulnicky 2006) have been studied in great detail, but other examples at similar distances have largely been neglected. IC 4662 (IBm) is one such case. It has a SMClike metallicity of $\log (\mathrm{O} / \mathrm{H})+12 \sim 8.1$, possesses two giant $\mathrm{HII}$ regions (A1 and $\mathrm{A} 2$ ) which dominate its visual appearance (Heydari-Malayeri et al. 1990), and lies at a distance of $2.44 \mathrm{Mpc}$ (Karachentsev et al. 2006). These H II regions host Wolf-Rayet (WR) populations (Rosa \& Dodorico 1986; Heydari-Malayeri et al. 1990; Richter \& Rosa 1991), indicating the presence of $\sim 5$ Myr old stellar populations, such that IC 4662 is one of the closest examples of a WR galaxy (Schaerer et al. 1999). However the spatial location and number of WR stars remains uncertain. Wolf-Rayet stars in metal-poor galaxies such

\footnotetext{
* Based on observations made with ESO telescopes at the Paranal observatory under program ID 65.H-0705(A) and archival NASA/ESA Hubble Space Telescope datasets, obtained from the ESO/ST-ECF Science Archive Facility.
}

as IC 4662 are believed to be the leading candidates for the progenitors of Type Ic supernova and some long duration Gamma Ray Bursts (GRBs) (Hammer et al. 2006; Modjaz et al. 2008).

Johnson et al. (2003) have proposed that IC 4662 contains deeply embedded star forming regions - referred to as ultradense $\mathrm{H}$ II regions - on the basis of radio observations. Such regions are typically very young and represent scaled-up versions of ultracompact H II regions. Such regions become optically visible within $\leq 0.5-1$ Myr according to Spitzer observations of $\mathrm{H}$ II regions within nearby star-forming galaxies (Prescott et al. 2007). If $\mathrm{A} 1$ and $\mathrm{A} 2$ were dominated by very young stellar populations, it would be surprising that $\sim 5 \mathrm{Myr}$ old WR stars are present. Hunter et al. (2001) have presented Infrared Space Observatory (ISO) imaging and spectroscopy of IC 4662, while Gilbert \& Vacca (2009) discuss Spitzer imaging and spectroscopy of these regions, supporting the claim of deeply embedded ionizing clusters by Johnson et al. (2003). McQuinn et al. (2009) have analysed Hubble Space Telescope (HST) imaging of IC 4662 from which they have estimated its recent star formation history.

In this paper we use Very Large Telescope (VLT) optical imaging and spectroscopy, supplemented by archival HST imaging to investigate the young, massive stellar content of IC 4662. This paper is organised as follows: VLT optical imaging and spectroscopy of IC 4662, and archival HST imaging are presented in Sect. 2. A nebular analysis is presented in Sect. 3, followed by an analysis of the massive stellar content of IC 4662 in Sect. 4. A discussion of the optical versus radio-derived properties of the giant H II regions is presented in Sect. 5, together with 
a comparison of the star formation rate (SFR) of IC 4662 with other nearby metal-poor star forming galaxies. Brief conclusions are reached in Sect. 6.

\section{Observations and data reduction}

Imaging and spectroscopy were obtained with the European Southern Observatory (ESO) VLT in September 2000, using the Focal Reduced/Low dispersion Spectrograph \#2 (FORS2). These datasets were supplemented by archival HST, Advanced Camera for Surveys (ACS) optical imaging. We follow the nomenclature of Heydari-Malayeri et al. (1990) for the four main $\mathrm{H}$ II regions of IC 4662, A, B, C and D throughout.

\subsection{VLT/FORS2 and CTIO $0.9 \mathrm{~m}$ imaging}

In the absence of our own ground-based $\mathrm{H} \alpha$ imaging of IC 4662, we have been kindly provided with a continuum-subtracted $\mathrm{H} \alpha+[\mathrm{N}$ II $]$ wide field image of IC 4662 obtained with the CTIO $0.9 \mathrm{~m}$ telescope on 14 Sep. 2001 (J. Lee, priv. comm.) See Kennicutt et al. (2008) for further details of these observations. This image is presented in the upper panel of Fig. 1. In addition to the main giant $\mathrm{H}$ II region complex $\mathrm{A}$, the figure highlights the fainter $\mathrm{H}$ II regions $\mathrm{B}$ and $\mathrm{C}$ to the south west and west of A, plus region $\mathrm{D}$, located 90 arcsec to the south east. The latter has previously been discussed by Hunter et al. (2001, referred to as IC 4662-A, therein). The published $\mathrm{H} \alpha$ flux of IC 4662 from Kennicutt et al. (2008) includes regions A-D, of which A1+A2 provide $\sim 70 \%$ of the total $\mathrm{H} \alpha$ emission.

A Bessell $V$-band $\left(\lambda_{\mathrm{c}}=554 \mathrm{~nm}, \Delta \lambda=111 \mathrm{~nm}\right)$ image of IC 4662 was obtained with VLT/FORS2 on 4 Sep. 2000 with an exposure time of $120 \mathrm{~s}$, during moderate seeing conditions $(\sim 0.75$ arcsec, airmass $\sim 1.4)$. The high resolution collimator provided a field of view of $3.4 \times 3.4$ arcmin $(0.1$ arcsec $\left.\mathrm{pix}^{-1}\right)$. The detector was a single $2048 \times 2048$ Tektronix CCD with $24 \mu \mathrm{m}$ pixels. A standard data reduction was applied, involving bias subtraction, using IRAF (Tody 1986). The central $25 \times 18$ arcsec region of IC 4662 , corresponding to $300 \times 220 \mathrm{pc}$, is presented in Fig. 2a, showing multiple sources within each of the giant $\mathrm{H}$ II regions $\mathrm{A} 1$ and $\mathrm{A} 2$ from Heydari-Malayeri et al. (1990).

In addition, interference filter imaging was obtained at the same time, centred upon the He II $\lambda 4686$ line $\left(\lambda_{\mathrm{c}}=469.1 \mathrm{~nm}\right.$, $\Delta \lambda=6.4 \mathrm{~nm})$ and the adjacent continuum $\left(\lambda_{\mathrm{c}}=478.9 \mathrm{~nm}\right.$, $\Delta \lambda=6.6 \mathrm{~nm}$ ). Exposure times were $600 \mathrm{~s}$ in both cases. The continuum subtracted He II image is presented in Fig. 2b, revealing two bright $\lambda 4686$ emission-line regions, one each within A1 and A2, plus two faint emission-line regions. One is $\sim 3 \operatorname{arcsec}$ to the west of the bright source in A2, while the other is offset $\sim 8$ arcsec north west from the bright source in A1. We refer to the latter source as A1-NW - previously the subject of a study by Richter \& Rosa (1991) - since it also hosts a 1 arcsec diameter H II region ${ }^{1}$.

\subsection{HST/ACS imaging}

We have retrieved high-resolution archival HST/ACS imaging of IC 4662 to supplement our ground-based datasets. Two separate programmes have obtained HST/ACS imaging, namely GO programme 10609 (PI W. D. Vacca) for which High Resolution

\footnotetext{
1 A1-NW is not spatially coincident with the compact radio continuum source IC 4662-N from Johnson et al. (2003) which lies 4 arcsec further to the east.
}

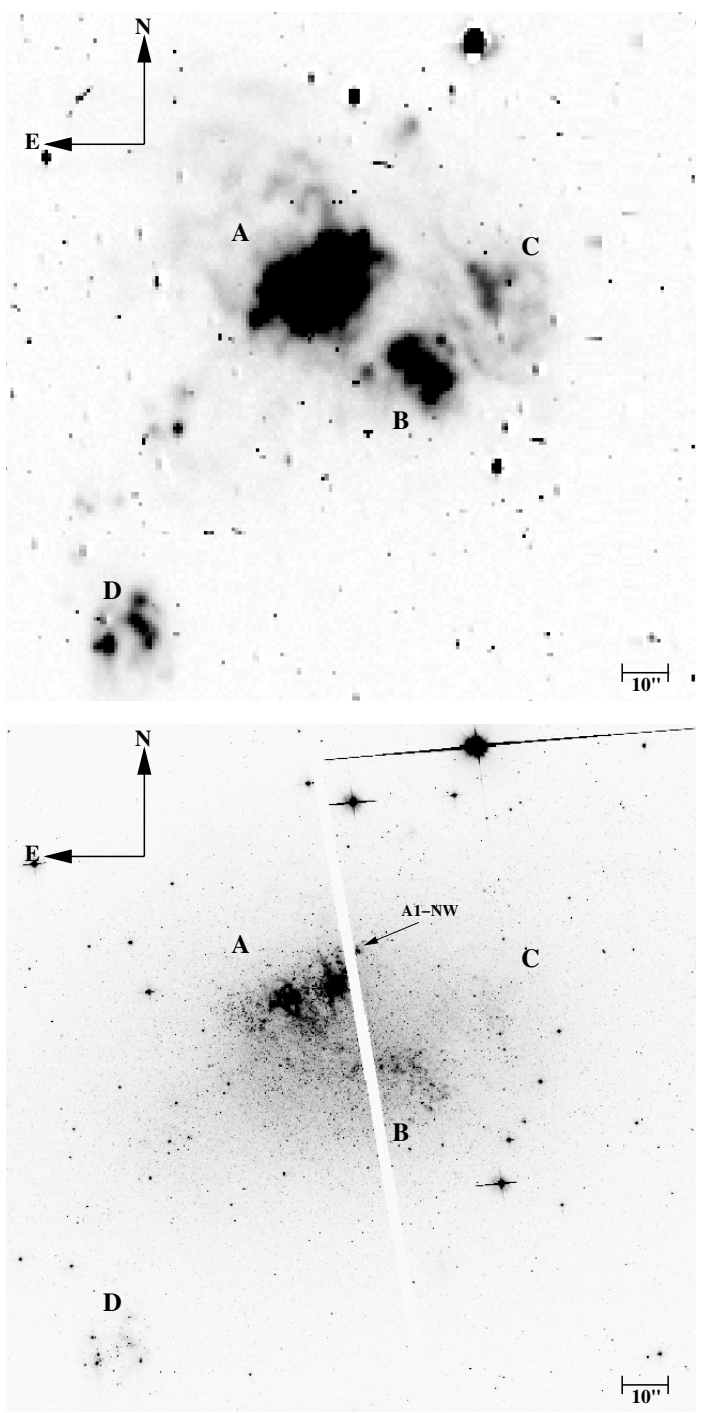

Fig. 1. $2.5 \times 2.5 \operatorname{arcmin}(1.8 \times 1.8 \mathrm{kpc}$ at $2.44 \mathrm{Mpc})$ image of IC 4662 from CTIO $0.9 \mathrm{~m}(\mathrm{H} \alpha+[\mathrm{N}$ II $]$, upper, see Kennicutt et al. 2008) and HST ACS/WFC (F606W, lower, see Karachentsev et al. 2006).

Camera (HRC) imaging (0.03 $\left.\operatorname{arcsec} \mathrm{pix}^{-1}\right)$ were obtained using the $F 330 W, F 435 W, F 550 M, F 658 N$ and $F 814 W$ filters with exposure times between 400-2000 s. In addition, ACS Wide Field Camera (WFC) imaging ( $\left.0.1 \operatorname{arcsec} \mathrm{pix}^{-1}\right)$ has been obtained under GO programme 9771 (PI I. D. Karachentsev) using the F606W (1200 s) and F814W (900 s) filters (Karachentsev et al. 2006).

In the lower panel of Fig. 1, we present the ACS/WFC F606W image of IC 4662 on the same scale as the ground-based CTIO $\mathrm{H} \alpha+[\mathrm{N} \mathrm{II}]$ image, which reveals that stellar populations in region $\mathrm{D}$ are physically separated from the remainder of the galaxy. Coordinates of individual sources were obtained from this ACS/WFC image, using a fit to 7 nearby GSC-2 reference stars in the STARLINK image analysis package GAIA. Johnson et al. (2003) have previously presented archival HST/WFPC2 F300W imaging of the central region of IC 4662.

In Fig. 2c we present the ACS/HRC F550M image of the central region of IC 4662, sampling the same region as that presented in Fig. 2a from VLT/FORS2. The improvement in spatial resolution allows us to identify individual He II $\lambda 4686$ excess regions from the FORS2 datasets, two within A1 that we shall hereafter refer to as A1-WR1 and A1-WR2 - the latter 0.4 arcsec 

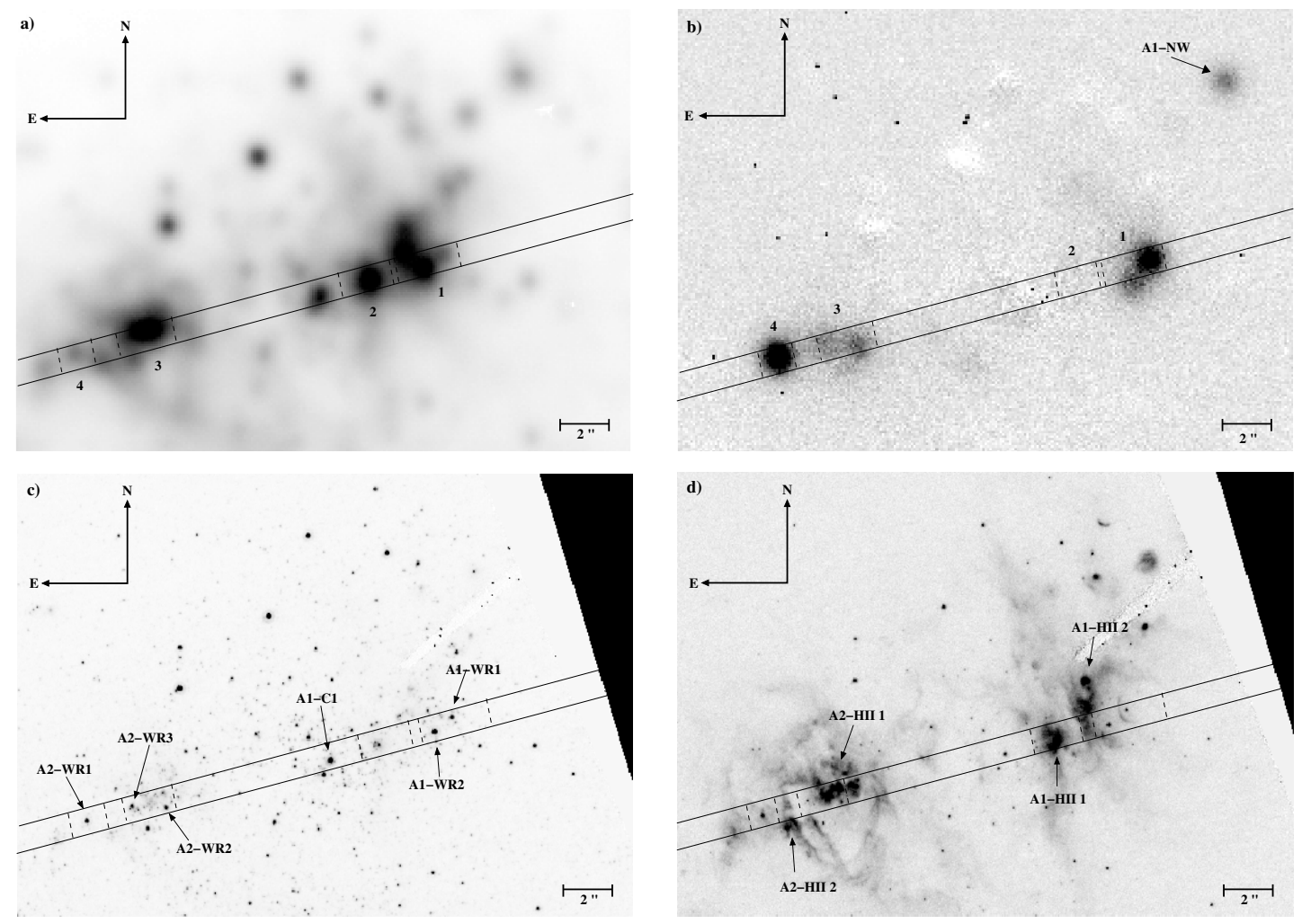

Fig. 2. VLT/FORS2 Bessell $V$-band (panel a)) and He II $\lambda 4686$ excess (panel b)) images of the central $25 \times 18$ arcsec $(300 \times 220 \mathrm{pc}$ at $2.44 \mathrm{Mpc})$ of IC 4662. The FORS2 long-slit has been overlaid, with extraction regions \#1 and 2 (within giant H II A1) and regions \#3 and 4 (within A2) marked. Archival HST/ACS high resolution camera images of the same region are presented in panel c) $(F 550 M)$ and d (F658N), whose footprint excluded the North West region A1-NW. A number of continuum sources and H II regions are indicated (see Sect. 2.2).

south of a much brighter source - plus three regions within A2, namely A2-WR1, A2-WR2 and A2-WR3. Sources A1-WR1, A1-WR2 and A2-WR1 are point-like, whereas sources A2-WR2 and A2-WR3 are spatially extended.

It is apparent from Fig. 2c that the He II $\lambda 4686$ emission region, A1-NW, to the north west does not lie within the footprint of the HRC datasets. Fortunately, it is included within the WFC imaging (Fig. 1, lower panel), revealing two point-like sources $\sim 0.2 \operatorname{arcsec}(\sim 2.5 \mathrm{pc})$ apart, located at the centre of extended $\mathrm{H} \alpha$ emission. Either (or both) of these may contribute to the $\lambda 4686$ emission. The visually brighter, south-west component is referred to as A1-WR3, with the fainter, north-east source labelled as A1-WR4.

In addition, we have labelled a bright, extended continuum source to the east of region A1 as A1-C1. This was included within our VLT/FORS2 long-slit, and allows us to absolutely flux calibrate our spectroscopy (see Sect. 2.3).

Finally, we present the HRC/F658N image of the central region of IC 4662 in Fig. 2d. Two bright knots of $\mathrm{H} \alpha$ emission can be seen within each of $\mathrm{A} 1$ and $\mathrm{A} 2$, that we shall refer to as A1-HII 1, A1-HII 2, A2-HII 1 and A2-HII 2, respectively. $3 \mathrm{~cm}$ peak intensities from Johnson et al. (2003) correspond to A1-HII 2 and A2-HII 1. Their continuum source IC4662-N corresponds to a faint arc located $6 \operatorname{arcsec}(\sim 75 \mathrm{pc})$ to the north of A1-HII 2 in Fig. 2 d.

\subsection{VLT/FORS2 spectroscopy}

VLT/FORS2 long slit spectroscopy was also carried out on 4 Sep. 2000, using the high resolution collimator and 600B grism, centred at $465 \mathrm{~nm}$. From the He II $\lambda 4686$ excess image
(Fig. 2b) we chose a position angle of 104 degrees east of north, to sample the two bright sources within A1 and A2, which also permitted the fainter source within A2 to be observed, though not A1-NW. A single $1800 \mathrm{~s}$ exposure was obtained using a 1 arcsec slit, albeit during observing conditions that had deteriorated considerably both in airmass $(\sim 2)$ and seeing ( $\sim 2 \operatorname{arcsec})$.

In total, four apertures were extracted, as shown in Fig. 2. Aperture 1 was centred upon the bright $\lambda 4686$ source in A1 (A1-WR1 plus A1-WR2 to its south-east), aperture 2 included the bright $\mathrm{H}$ II region (A1-HII 1), aperture 3 included the fainter $\lambda 4686$ source in A2 (A2-WR2 and A2-WR3 to its east) and finally aperture 4 included the bright $\lambda 4686$ source in A2 (A2-WR1). Due to the poor quality of the observing conditions, we also extracted the region centred upon the continuum source A1-C1, in order to facilitate absolute flux calibration.

A standard data reduction followed, involving bias subtraction and extraction of apertures using IRAF. Wavelength calibration was achieved using a reference $\mathrm{He} / \mathrm{Hg} / \mathrm{Cd}$ arc lamp, revealing a spectral range of 3710-6170 A, plus a spectral resolution of $7 \AA$ as measured from arc lines. Unfortunately, the primary spectrophotometric standard star was observed using a different central wavelength to the target, so a secondary star, HD 5980, observed during the same night with the same setup as IC 4662 was used in its place. An unpublished, flux calibrated dataset of HD 5980 obtained with the Mt Stromlo 2.3 m Dual Beam Spectrograph (DBS) in Dec. 1997 enabled a relative flux calibration.

Absolute flux calibration for each aperture was achieved in two steps, given the non-photometric conditions of the spectroscopic datasets. First, the continuum flux of the aperture centred upon A1-C1 was set to the average of its $F 435 \mathrm{~W}$ and F550M 
Table 1. Observed line fluxes, $F_{\lambda}$, and intensities, $I_{\lambda}$, of nebular and stellar (Wolf-Rayet, WR) lines in each of the four VLT/FORS2 IC 4662 apertures, relative to $\mathrm{H} \beta=100 \mathrm{H} \beta$ fluxes are shown separate from normalised values in units of $10^{-13} \mathrm{erg} \mathrm{s}^{-1} \mathrm{~cm}^{-2}$.

\begin{tabular}{|c|c|c|c|c|c|c|c|c|c|c|}
\hline \multirow[t]{2}{*}{$\lambda(\AA)$} & \multicolumn{2}{|l|}{ ID } & \multicolumn{2}{|r|}{ \#1 } & \multicolumn{2}{|c|}{ \#2 } & \multicolumn{2}{|c|}{ \#3 } & \multicolumn{2}{|c|}{ \#4 } \\
\hline & & & $F_{\lambda}$ & $I_{\lambda}$ & $F_{\lambda}$ & $I_{\lambda}$ & $F_{\lambda}$ & $I_{\lambda}$ & $F_{\lambda}$ & $I_{\lambda}$ \\
\hline 3727 & [O II] & & 66.2 & 83.8 & 102.2 & 113.9 & 146.7 & 174.8 & 142.2 & 163.6 \\
\hline 3867 & [Ne III] & & 40.6 & 50.1 & 57.6 & 62.6 & 10.1 & 11.8 & 183.6 & 204.7 \\
\hline 3968 & {$[\mathrm{Ne}$ III $]+\mathrm{H} 7$} & & 24.8 & 30.1 & 22.4 & 24.2 & 6.2 & 7.1 & 115.0 & 127.1 \\
\hline 4058 & NIV & WR & 1.8 & 2.1 & - & - & 1.8 & 2.1 & 0.5 & 0.6 \\
\hline 4100 & $\mathrm{H} \delta$ & & 21.1 & 25.4 & 23.9 & 27.1 & 22.9 & 26.4 & 25.0 & 28.5 \\
\hline 4340 & $\mathrm{H} \gamma$ & & 41.4 & 46.4 & 45.9 & 49.9 & 42.3 & 45.5 & 42.8 & 46.4 \\
\hline 4363 & [O III] & & 7.1 & 7.9 & 6.4 & 7.4 & 6.4 & 7.0 & 6.8 & 7.4 \\
\hline 4659 & [Fe III] & & 0.41 & 0.43 & 0.47 & 0.49 & 6.3 & 6.5 & 0.57 & 0.59 \\
\hline $4686^{*}$ & He II & WR & 8.5 & 8.9 & 2.1 & 2.2 & 4.17 & 4.25 & 14.6 & 15.3 \\
\hline 4712 & [Ar IV] & & 2.0 & 2.1 & 1.5 & 1.5 & 0.96 & 0.99 & 1.2 & 1.2 \\
\hline 4741 & [Ar IV] & & 0.96 & 0.99 & 0.70 & 0.72 & 0.36 & 0.37 & 0.54 & 0.56 \\
\hline 4861 & $\mathrm{H} \beta$ & & 100 & 100 & 100 & 100 & 100 & 100 & 100 & 100 \\
\hline 4959 & [O III $]$ & & 213.8 & 206.3 & 198.4 & 198.5 & 184.8 & 180.4 & 203.6 & 202.0 \\
\hline 5007 & [O III] & & 630.0 & 604.0 & 583.0 & 590.6 & 542.9 & 528.6 & 604.4 & 607.8 \\
\hline 5411 & He II & WR & 0.56 & 0.50 & - & - & - & - & 1.16 & 1.15 \\
\hline 5808 & C IV & WR & - & - & - & - & 2.7 & 2.4 & - & - \\
\hline 5876 & $\mathrm{He} \mathrm{I}$ & & 12.1 & 9.8 & 11.7 & 11.3 & 13.9 & 12.0 & 11.9 & 11.1 \\
\hline $\mathrm{H} \beta$ & & & 0.44 & 1.12 & 0.39 & 0.49 & 0.44 & 0.86 & 0.13 & 0.20 \\
\hline \multicolumn{2}{|c|}{$E(B-V)$} & & \multicolumn{2}{|c|}{$0.28 \pm 0.05$} & \multicolumn{2}{|c|}{$0.08 \pm 0.05$} & \multicolumn{2}{|c|}{$0.20 \pm 0.05$} & \multicolumn{2}{|c|}{$0.12 \pm 0.05$} \\
\hline \multicolumn{2}{|c|}{$T_{\mathrm{e}}(\mathrm{K})$} & & \multicolumn{2}{|c|}{$12900 \pm 700$} & \multicolumn{2}{|c|}{$12700 \pm 900$} & \multicolumn{2}{|c|}{$12900 \pm 700$} & \multicolumn{2}{|c|}{$12500 \pm 400$} \\
\hline \multicolumn{2}{|c|}{$\mathrm{O}^{+} / \mathrm{H}\left(\times 10^{-5}\right)$} & & \multicolumn{2}{|c|}{$1.20 \pm 0.07$} & \multicolumn{2}{|c|}{$1.7 \pm 0.1$} & \multicolumn{2}{|c|}{$2.5 \pm 0.18$} & \multicolumn{2}{|c|}{$2.6 \pm 0.2$} \\
\hline \multirow{2}{*}{\multicolumn{2}{|c|}{$\begin{array}{l}\mathrm{O}^{2+} / \mathrm{H}\left(\times 10^{-5}\right) \\
12+\log (\mathrm{O} / \mathrm{H})\end{array}$}} & & \multicolumn{2}{|c|}{$10.3 \pm 0.53$} & \multicolumn{2}{|c|}{$10.6 \pm 0.61$} & \multicolumn{2}{|c|}{$9.0 \pm 0.65$} & \multicolumn{2}{|c|}{$11.2 \pm 0.87$} \\
\hline & & $12+\log (\mathrm{O} / \mathrm{H})$ & \multicolumn{2}{|c|}{$8.06 \pm 0.02$} & \multicolumn{2}{|c|}{$8.09 \pm 0.02$} & \multicolumn{2}{|c|}{$8.06 \pm 0.03$} & \multicolumn{2}{|c|}{$8.14 \pm 0.03$} \\
\hline
\end{tabular}

${ }^{*}$ He II $\lambda 4686$ fluxes are the sum of nebular and stellar components.

fluxes, which were obtained from photometry using DAOPHOT, the point-spread function (PSF) fitting routine within IRAF. The resulting flux calibrated spectrum agreed with the photometry to within $10 \%$, but of course relies upon A1-C1 dominating the VLT/FORS2 aperture. Second, slit losses of the other apertures (with respect to A1-C1) were estimated from the two dimensional spectral image, indicating throughputs of $60-85 \%$ parallel to the slit, and adopting a uniform $50 \%$ transmission perpendicular to the slit. Overall, agreement between the flux calibrated spectra and the flux inferred from bright sources within each aperture was satisfactory, suggesting errors of $\sim 25 \%$.

\section{Nebular analysis}

In this section we shall derive the nebular properties of IC 4662 A1 and A2 from VLT/FORS2 spectroscopy and HST/ACS F658N imaging, and present comparisons with previous results.

\subsection{Properties of $A 1$ and $A 2$ from spectroscopy}

Our analysis was performed using Gaussian fits to the observed high excitation nebular (and stellar) emission lines using the Emission Line Fitting (ELF) routine within the STARLINK package DIPSO. These are presented in Table 1.

The interstellar extinction for each aperture was determined using the observed Balmer line ratios $F(\mathrm{H} \gamma) / F(\mathrm{H} \beta)$ and $F(\mathrm{H} \delta) / F(\mathrm{H} \beta)$ together with Case B recombination theory (Hummer \& Storey 1987), assuming $n_{\mathrm{e}} \sim 100 \mathrm{~cm}^{-3}$ and $T_{\mathrm{e}} \sim$ $10^{4} \mathrm{~K}$, plus a standard Galactic extinction law (Seaton 1979). Extinctions ranged from $0.08 \mathrm{mag}$, just above the foreground extinction of $E(B-V)=0.07 \mathrm{mag}$ (Schlegel et al. 1998), to $0.28 \mathrm{mag}$, with average values of $E(B-V)=0.162$ and 0.165 mag for regions $\mathrm{A} 1$ and $\mathrm{A} 2$, respectively. We assign an uncertainty of \pm 0.05 to these values, given problems associated

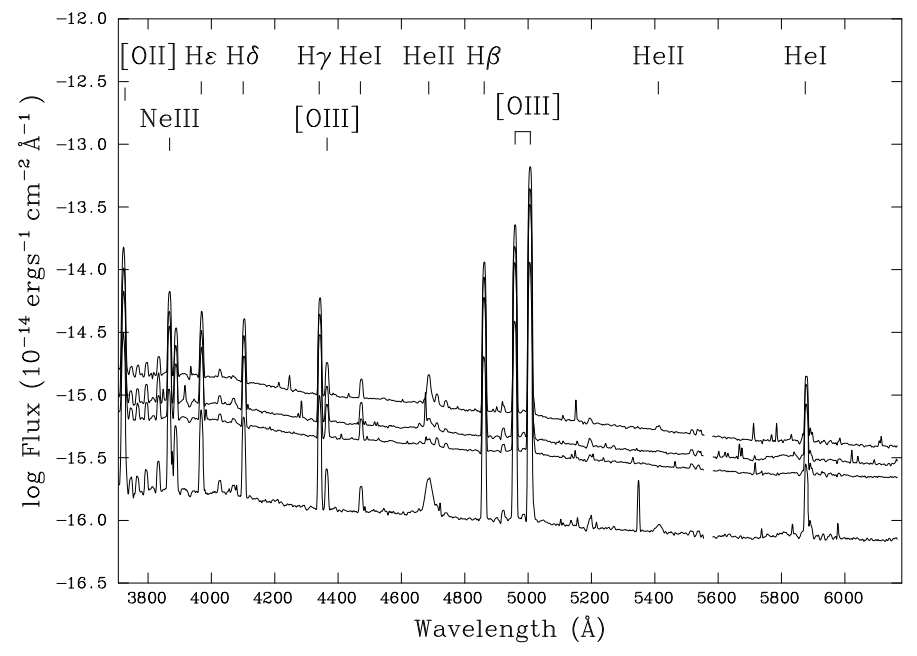

Fig. 3. Flux calibrated, dereddened VLT/FORS2 spectra of IC 4662 apertures \#1, 3, 2 and 4 (from top to bottom).

with flux calibration and the neglect of underlying (stellar) absorption lines. Consequently, these are consistent - within the quoted errors - with values of 0.11 and 0.14 mag for A1 and A2 from Hidalgo-Gámez et al. (2001). McQuinn et al. (2009) estimated an extinction of $A_{\mathrm{V}} \approx 3.1 E(B-V) \approx 0.3-0.4$ towards IC 4662 from their analysis of HST/ACS imaging.

We present the de-reddened spectra of apertures \#1-4 in Fig. 3. In spite of the low spectral resolution, strong [O III] $\lambda 4363$ emission is observed in all cases, allowing a determination of the nebular temperature. Emission line fluxes and intensities are presented in Table 1, including both strong nebular and stellar lines, the latter indicated with "WR", although He II $\lambda 4686$ may be of stellar, or nebular, origin (see Sect. 4.2). 
Table 2. Observed $\mathrm{H} \alpha$ fluxes of IC4662 A1 and A2 from optical HST/F658N imaging (6 arcsec radii), compared to radio and mid-IR results.

\begin{tabular}{lccc}
\hline \hline Quantity & Units & A1 & A2 \\
\hline$F(\mathrm{H} \alpha+[\mathrm{N} \mathrm{II}])$ & $\left(\mathrm{erg} \mathrm{s}^{-1} \mathrm{~cm}^{-2}\right)$ & $1.94 \times 10^{-12}$ & $1.61 \times 10^{-12}$ \\
$F[\mathrm{~N} \mathrm{II}] / F(\mathrm{H} \alpha)$ & & 0.03 & 0.03 \\
$F(\mathrm{H} \alpha)$ & $\left(\mathrm{erg} \mathrm{s}^{-1} \mathrm{~cm}^{-2}\right)$ & $1.89 \times 10^{-12}$ & $1.56 \times 10^{-12}$ \\
$E(B-V)$ & $\left(\mathrm{mag}^{-}\right.$ & 0.162 & 0.165 \\
$I(\mathrm{H} \alpha)$ & $\left(\mathrm{erg} \mathrm{s}^{-1} \mathrm{~cm}^{-2}\right)$ & $2.75 \times 10^{-12}$ & $2.30 \times 10^{-12}$ \\
$L(\mathrm{H} \alpha)$ & $\left(\mathrm{erg} \mathrm{s}^{-1}\right)$ & $1.96 \times 10^{39}$ & $1.63 \times 10^{39}$ \\
$Q_{0}(\mathrm{H} \alpha)$ & $\left(10^{49} \mathrm{~s}^{-1}\right)$ & 145 & 121 \\
\hline$Q_{0}(3 \mathrm{~cm})^{*}$ & $\left(10^{49} \mathrm{~s}^{-1}\right)$ & 227 & 242 \\
$Q_{0}(\mathrm{Hu} \alpha) \neq$ & $\left(10^{49} \mathrm{~s}^{-1}\right)$ & 345 & 256 \\
\hline
\end{tabular}

* Johnson et al. (2003) adjusted to $2.44 \mathrm{Mpc}$.

$¥$ Gilbert \& Vacca (2009) adjusted to $2.44 \mathrm{Mpc}$.

Using the $[\mathrm{O}$ III $](4959+5007) / 4363 \AA$ line intensity ratio (Osterbrock \& Ferland 2006) we calculate $T_{\mathrm{e}}$ for each region, for which we find $T_{\mathrm{e}} \sim 12700 \mathrm{~K}$ in each case (Table 1), similar to $T_{\mathrm{e}} \sim 12000 \mathrm{~K}$ from Hidalgo-Gámez et al. (2001). Unfortunately, our VLT/FORS2 spectroscopy is of insufficient resolution to separate the [O II] $\lambda 3726,3729$ doublet and does not cover the [S II] $\lambda 6717,6731$ doublet, so we adopt a typical H II region electron density of $\sim 100 \mathrm{~cm}^{-2}$. For comparison, Gilbert \& Vacca (2009) obtained $n_{\mathrm{e}}=150(212) \mathrm{cm}^{-2}$ for A1 (A2) from the observed [S III] $18.7 \mu \mathrm{m} / 33.5 \mu \mathrm{m}$ ratio in their Spitzer/IRS datasets. Emissivities were then determined for each aperture using FIVEL (De Robertis et al. 1987), from which the oxygen abundance was calculated in each case. We find an average metallicity of $12+\log (\mathrm{O} / \mathrm{H}) \sim 8.1$, close to that of the SMC (Russell \& Dopita 1990), and in excellent agreement with the previous determinations of $\mathrm{A} 1$ and $\mathrm{A} 2$ by Heydari-Malayeri et al. (1990) and Hidalgo-Gámez et al. (2001). Oxygen abundances for individual apertures are also presented in Table 1 . These are consistent with metal contents of $0.12-$ $0.17 \times$ solar for neon and sulphur obtained by Gilbert \& Vacca (2009) from Spitzer/IRS spectroscopy of A1 and A2. Note that Hidalgo-Gámez et al. (2001) obtain a substantially lower oxygen abundance of $\log (\mathrm{O} / \mathrm{H})+12=7.7$ for region $\mathrm{D}$.

\subsection{Properties of $A 1$ and $A 2$ from imaging}

The ionizing fluxes of giant H II regions IC 4662-A1 and A2 may be estimated from their $\mathrm{H} \alpha$ luminosity (Kennicutt et al. 2008). Aperture photometry of the ACS/HRC F658N image (recall Fig. 2d) allows the $\mathrm{H} \alpha+[\mathrm{N}$ II] fluxes of A1 and A2 to be measured, using radii of $6.15 \operatorname{arcsec}(75 \mathrm{pc})$ and $5.5 \operatorname{arcsec}(65 \mathrm{pc})$, respectively, under the reasonable assumption that the stellar contribution to this filter is negligible. We defer a discussion of the global star formation rate of IC 4662 until Sect. 5.

Table 2 presents observed $\mathrm{H} \alpha$ fluxes for these regions, corrected for both background and aperture size (Sirianni et al. 2005). We also account for the contribution of [N II] to the $F 658 N$ flux using the ratio $F[\mathrm{~N} \mathrm{II}] / F(\mathrm{H} \alpha) \sim 0.03$ obtained by Hidalgo-Gámez et al. (2001). For our adopted distance of $2.44 \mathrm{Mpc}$, the Kennicutt (1998) conversion from $\mathrm{H} \alpha$ luminosity to ionizing flux, $Q_{0}$, implies $145 \times 10^{49} \mathrm{~s}^{-1}$ and $121 \times 10^{49} \mathrm{~s}^{-1}$ for A1 and A2, respectively. This equates to 145 and 121 equivalent O7V stars, based upon an ionizing flux of $\sim 10^{49}$ photon $^{-1}$ for SMC-metallicity O7V stars (Hadfield \& Crowther 2006).

In Table 2 we compare these values to those obtained from $3 \mathrm{~cm} / 8.6 \mathrm{GHz}$ continuum fluxes (Johnson et al. 2003), and $\mathrm{Hu} \alpha$
Table 3. VLT/FORS2 nebular and stellar properties of apertures 1-4 within IC4662 A1 and A2, including estimated $\mathrm{O}$ star numbers, $N(\mathrm{O})$, and luminosities of nebular and Wolf-Rayet lines.

\begin{tabular}{lccccc}
\hline \hline & & \multicolumn{2}{c}{$\mathrm{A} 1$} & \multicolumn{2}{c}{ A2 } \\
& Units & $\# 1$ & $\# 2$ & $\# 3$ & $\# 4$ \\
\hline$L(\mathrm{H} \beta)$ & $\left(\mathrm{erg} \mathrm{s}^{-1}\right)$ & $8.0 \times 10^{37}$ & $3.5 \times 10^{37}$ & $6.2 \times 10^{37}$ & $1.4 \times 10^{37}$ \\
$\log Q_{0}$ & $\left(\right.$ photon s$\left.^{-1}\right)$ & 50.22 & 49.87 & 50.11 & 49.47 \\
$W_{\lambda}(\mathrm{H} \beta)$ & $(\AA)$ & 138 & 159 & 177 & 197 \\
$\eta_{0}$ & & 0.2 & 0.25 & 0.25 & 0.25 \\
$N(\mathrm{O})$ & & 84 & 30 & 52 & 12 \\
$L\left(\mathrm{He}\right.$ II $\left.^{\mathrm{WR}} 4686\right)$ & $\left(\mathrm{erg} \mathrm{s}^{-1}\right)$ & $4.4 \times 10^{36}$ & - & $2.1 \times 10^{36}$ & $1.8 \times 10^{36}$ \\
$L\left(\mathrm{He}\right.$ II $\left.^{\text {neb }} 4686\right)$ & $\left(\mathrm{erg} \mathrm{s}^{-1}\right)$ & $2.7 \times 10^{36}$ & $5.3 \times 10^{35}$ & $3.0 \times 10^{35}$ & - \\
$L(\mathrm{C}$ IV 5801 $)$ & $\left(\mathrm{erg} \mathrm{s}^{-1}\right)$ & - & - & $\leq 1.5 \times 10^{36}$ & - \\
\hline
\end{tabular}

$(12.4 \mu \mathrm{m})$ line fluxes from Spitzer/IRS spectroscopy (Gilbert \& Vacca 2009). The optically derived ionizing flux of A1 is approximately $2 / 3$ of the radio derived value, while that of A2 is approximately $1 / 2$ of the $3 \mathrm{~cm}$ determination, supporting previous suggestions of deeply embedded sources within A1 and A2, albeit contributing at most 30-50\% of the total ionizing flux.

In A1, the Spitzer/IRS derived Lyman continuum flux is apparently higher than that from the radio continuum. However, the $\mathrm{Hu} \alpha$ line appears unusually broad in the IRS dataset with respect to other nebular lines, suggesting a blend with another feature, which could account for up to $50 \%$ of the observed $\mathrm{Hu} \alpha$ flux, bringing it closer into line with value obtained from $\mathrm{H} \alpha$ (W. D. Vacca, priv. comm.).

\section{Analysis of individual sources}

In this section we use our VLT/FORS2 spectroscopy to estimate the massive star content of each region, supplemented by narrow-band imaging datasets for region A1-NW which was not observed spectroscopically. We also infer properties of individual ACS/HRC and WFC sources, for which slit spectroscopy is available.

\subsection{O star content}

In the previous section we have obtained estimates of the equivalent number of O7V stars for A1 and A2 from ACS/HRC $\mathrm{H} \alpha$ imaging. In reality, the $\mathrm{O}$ star content of individual regions of IC 4662 depends upon the age via

$N(\mathrm{O})=\frac{N_{\mathrm{O} 7 \mathrm{~V}}}{\eta_{0}}$

where $\eta_{0}$ is a sensitive function of age (Vacca 1994; Schaerer \& Vacca 1998). Ages can be estimated from the equivalent width of Balmer lines, such as $\mathrm{H} \beta$ in our VLT/FORS2 spectra. Table 3 presents the measured $W_{\lambda}(\mathrm{H} \beta)$ for each of our four apertures. Combining individual apertures reveals $W_{\lambda}(\mathrm{H} \beta) \sim 150 \AA$ and $190 \AA$ for A1 and A2, respectively. Slit spectroscopy of these regions by Heydari-Malayeri et al. (1990) revealed lower equivalent widths of 146 and $122 \AA$, using a 2 arcsec slit width.

From SMC-metallicity evolutionary synthesis models (Schaerer \& Vacca 1998), such line strengths suggest characteristic ages of 4.7 and 4.5 Myr for the regions sampled within A1 and A2, respectively, neglecting the minor contribution of weak stellar $\mathrm{H} \beta$ absorption. In reality, young clusters will only contribute a fraction of the total continuum light sampled by the ground-based VLT/FORS2 spectroscopy, so the actual ages of clusters are likely to be somewhat younger than that inferred. 


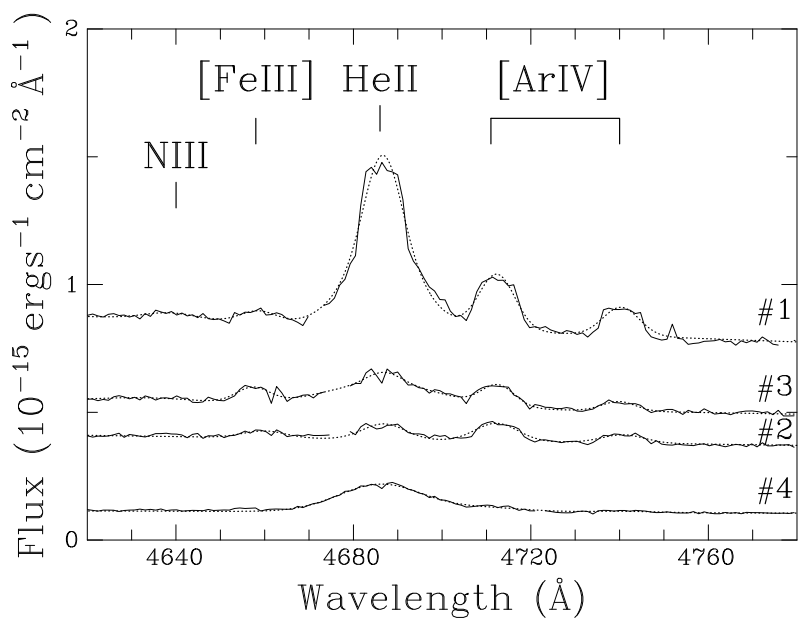

Fig. 4. De-reddened VLT/FOR2 spectroscopy of apertures $1-4$ in the vicinity of He II $\lambda 4686$ revealing both nebular and stellar components, plus nebular [Fe III] $\lambda 4658$ and [Ar IV] $\lambda 4711,4741$ (Gaussian fits are shown as dotted lines).

Nevertheless, $\eta_{0} \sim 0.2-0.25$ follows from the measured $\mathrm{H} \beta$ equivalent widths, allowing estimates of the number of $\mathrm{O}$ stars, $N(\mathrm{O})$, as presented in Table 3.

These estimates serve as upper limits since the contribution of Wolf-Rayet stars to ionizing fluxes are neglected, and are solely valid for the sub-regions of A1 and A2 that are sampled by the individual apertures (recall Fig. 2a). Therefore one should not infer global $\mathrm{O}$ star numbers for $\mathrm{A} 1$ and $\mathrm{A} 2$ from our age estimates, since there will doubtless be a spread in ages of sources within these regions. For $\mathrm{A} 1-\mathrm{C} 1$, a significantly smaller $\mathrm{H} \beta$ equivalent width of $\sim 48 \AA$ was measured, suggesting a greater age of $\sim 6 \mathrm{Myr}$.

\subsection{Wolf-Rayet populations}

In Fig. 4 we present spectroscopy of the four VLT/FORS2 apertures around the He II $\lambda 4686$ line. From comparison with adjacent [Fe III] and [Ar IV] nebular lines, the observed He II lines comprise (narrow) nebular and (broad) stellar components, the latter from Wolf-Rayet stars.

We can estimate the Wolf-Rayet populations within individual apertures from the stellar He II $\lambda 4686$ line luminosity, so we have taken care to distinguish between nebular and stellar components. Stellar line luminosities for regions \#1, \#3 and \#4, lie in the range $1.8-4.4 \times 10^{36} \mathrm{erg} \mathrm{s}^{-1}$, with $F W H M($ He II $) \approx 20$ $30 \AA$. N IV $\lambda 4058$ emission is observed in these regions, with $\mathrm{N}$ III $\lambda 4634-41$ weak or absent, suggesting mid-type nitrogen sequence (WN5-6) spectral types.

Crowther \& Hadfield (2006) present line luminosities for individual LMC and SMC WN5-6 stars. From a sample of 15 LMC WN5-6(ha) stars, the mean He II line luminosity is $1.75 \times 10^{36} \mathrm{erg} \mathrm{s}^{-1}$. Therefore, the observed line luminosities of \#1, \#3 and \#4 are each consistent with 1-2 luminous, hydrogen-rich WN5-6ha stars (Table 3). Alternatively, we also consider the possibility that sources are clusters hosting multiple hydrogen-poor WN stars. In view of the measured oxygen abundance for IC 4662, we would instead adopt the $4.3 \times$ $10^{35} \mathrm{erg} \mathrm{s}^{-1}$ mean of 2 SMC WN5-6 stars, from which 4-10 (classical) WN5-6 stars are inferred, omitting any contributions from WC stars.

Weak, broad CIV 15801-12 from early-type WC stars is observed solely in region \#3. Unfortunately, no WC4 stars are observed in the SMC, so we face the choice of adopting a mean $\mathrm{C}$ IV line luminosity of $3.3 \times 10^{36} \mathrm{erg} \mathrm{s}^{-1}$ from LMC WC4 stars, or that of the sole SMC WO star. O VI $13811-34$ does not appear to be present in our VLT/FORS2 spectroscopy, favouring a single WC4 star in region 3. The C IV FWHM is difficult to measure, since it is blended with weak [N II] $\lambda 5755$ emission, but supports a WC4 subtype since $F W H M \sim 80 \AA$. In addition, from the observed ratio of the C III $\lambda 4650+$ He II $\lambda 4686$ blend to the C IV $25801-12$ line luminosity in LMC WC4 stars, one would expect a contribution of $\sim 2 \times 10^{36} \mathrm{erg} \mathrm{s}^{-1}$ to the $\lambda 4650 / 4686$ line luminosity in region 3 from the WC4 star. This is similar to that observed, and would suggest that WN stars do not contribute to this region. However, the observed peak wavelength of the blue feature in region \#3 lies at $\lambda 4686$ rather than $\lambda 4650$, arguing against this interpretation. In fact, two separate sources are observed within region \#3, namely A2-WR2 and A2-WR3. One may host a WC star and the other a WN-type star.

In addition to stellar He II $14686, \# 1-3$ reveal nebular He II emission, with $I(\mathrm{He}$ II $) / I(H \beta)=0.03$ in region 1 and $\leq 0.01$ in regions $2-3$. Hidalgo-Gámez et al. (2001) obtained $I(\mathrm{He} \mathrm{II}) / I(\mathrm{H} \beta)=0.05$ for stellar and nebular 14686 . Other high ionization lines are observed in A1 and A2, namely [O IV] $25.9 \mu \mathrm{m}$ from Spitzer/IRS (Gilbert \& Vacca 2009). Since $\mathrm{O}$ stars are incapable of producing significant numbers of high energy photons, either shocks or Wolf-Rayet stars (Hadfield \& Crowther 2006) are likely to be responsible for the nebular He II and [OIV] lines. The former could be reconciled with the absence of WR stars in region 2 owing to spatially extended nebular He II emission from region 1.

Finally, although our VLT/FORS2 spectroscopy did not include the north west region of A1, we have estimated its line flux through the He II $\lambda 4686$ filter from a comparison with the spectroscopic line flux of region 4 (recall Fig. 2b). We obtain a He II line flux of $10^{-15} \mathrm{erg} \mathrm{s}^{-1} \mathrm{~cm}^{-2}$ for region A1-NW. Richter \& Rosa (1991) have published spectroscopy of this source, revealing an early-type WC subtype. Neglecting any potential nebular emission, for an assumed extinction of $E(B-V)=0.07 \mathrm{mag}$ we estimate a luminosity of $\approx 10^{36} \mathrm{erg} \mathrm{s}^{-1}$ in the $469.1 \mathrm{~nm}$ filter. For WC stars, approximately half of the C III $\lambda 4650 / \mathrm{He}$ II $\lambda 4686$ luminosity contributes to the $469.1 \mathrm{~nm}$ filter, so the total blue luminosity is $\approx 2 \times 10^{36} \mathrm{erg} \mathrm{s}^{-1}$, consistent with a sole WC star. We are unable to discriminate between the two potential sources (A1-WR3 and A1-WR4) from our ground-based imaging since they are only $0.2 \operatorname{arcsec}(\sim 2 \mathrm{pc})$ apart in ACS/WFC datasets and possess similar $m_{\mathrm{F} 606 \mathrm{~W}}-m_{\mathrm{F} 814 \mathrm{~W}}$ colours.

Our estimate of the stellar content of A1-NW is significantly lower than previous ESO $2.2 \mathrm{~m}$ narrow-band imaging and $3.6 \mathrm{~m} / \mathrm{IDS}$ spectroscopy of this region by Richter \& Rosa (1991). They obtained an integrated C IV $\lambda 4650 / \mathrm{He}$ II $\lambda 4686$ flux of $3 \times 10^{-14} \mathrm{erg} \mathrm{s}^{-1} \mathrm{~cm}^{-2}$, from which $\sim 10$ late WN and early WC stars were inferred. For a solely foreground extinction, their quoted nebular $\mathrm{H} \beta$ flux would imply $L(\mathrm{H} \beta)=1.1 \times 10^{38} \mathrm{erg} \mathrm{s}^{-1}$ or $Q_{0}=2.3 \times 10^{50}$ photon $\mathrm{s}^{-1}$. The reason for this substantial difference is unclear.

\subsection{Clusters or single stars?}

Our VLT/FORS2 imaging allows us to identify the location of $\lambda 4686$ emission line sources, plus spectroscopy allows crude estimates of the massive stellar content of individual apertures. However, recalling Fig. 2c, ACS/HRC imaging reveals multiple sources within these apertures. Such sources are either compact $(\sim \mathrm{pc})$ clusters or individual stars. 
Table 4. Vega magnitudes from ACS/HRC (ACS/WFC for A1-WR3 and A1-WR4) imaging, plus inferred cluster properties from STARBURST99 (Leitherer et al. 1999) models, for ages of $4.5 \mathrm{Myr}(\sim 6 \mathrm{Myr}$ for A1-C1).

\begin{tabular}{|c|c|c|c|c|c|c|c|c|c|}
\hline $\begin{array}{c}\text { Source } \\
\text { Aperture }\end{array}$ & & $\begin{array}{c}\text { A1-WR1 } \\
\# 1\end{array}$ & $\begin{array}{c}\text { A1-WR2 } \\
\# 1\end{array}$ & $\overline{\mathrm{A} 1-\mathrm{C} 1}$ & $\begin{array}{c}\text { A2-WR1 } \\
\# 4\end{array}$ & $\begin{array}{c}\text { A2-WR2 } \\
\# 3\end{array}$ & $\begin{array}{c}\text { A2-WR3 } \\
\# 3\end{array}$ & $\begin{array}{l}\text { A1-WR3 } \\
\text { (A1-NW) }\end{array}$ & $\begin{array}{l}\text { A1-WR4 } \\
\text { (A1-NW) }\end{array}$ \\
\hline RA (J2000) & & $17: 47: 08.66$ & $17: 47: 08.77$ & $17: 47: 09.45$ & $17: 47: 11.03$ & $17: 47: 10.52$ & $17: 47: 10.74$ & $17: 47: 08.18$ & $17: 47: 08.20$ \\
\hline $\operatorname{Dec}(\mathrm{J} 2000)$ & & $-64: 38: 17.1$ & $-64: 38: 18.1$ & $-64: 38: 18.8$ & $-64: 38: 21.2$ & $-64: 38: 20.6$ & $-64: 38: 20.6$ & $-64: 38: 09.8$ & $-64: 38: 09.7$ \\
\hline$m_{\mathrm{F} 550 \mathrm{M}}$ & (mag) & 19.53 & 20.82 & 18.72 & 19.25 & 19.85 & 19.97 & $20.17^{1}$ & $20.29^{1}$ \\
\hline$m_{\mathrm{F} 435 \mathrm{~W}}-m_{\mathrm{F} 550 \mathrm{M}}$ & (mag) & -0.18 & -0.35 & -0.18 & -0.18 & -0.09 & 0.16 & - & - \\
\hline$E(B-V)$ & (mag) & $(0.07)$ & $(0.07)$ & $(0.07)$ & $(0.07)$ & 0.08 & 0.33 & $(0.07)$ & $(0.07)$ \\
\hline $\begin{array}{c}M_{\mathrm{F} 550 \mathrm{M}} \\
\text { Mass }\end{array}$ & (mag) & -7.6 & -6.3 & $\begin{array}{c}-8.4 \\
3.4\end{array}$ & -7.9 & $\begin{array}{c}-7.3 \\
1.3\end{array}$ & -7.9 & $-7.0^{1}$ & $-6.9^{1}$ \\
\hline WR? & () & $1-2 \times$ WN5-6ha & WN5-6? & - & $1-2 \times$ WN5-6ha & WN5-6 or WC & WN5-6 or WC & WC? & WC? \\
\hline
\end{tabular}

Note: adopted interstellar extinctions are shown in parenthesis.

${ }^{1} m_{\mathrm{F} 606 \mathrm{~W}}$.

We performed photometry of ACS/HRC F435W and F550M imaging with DAOPHOT to obtain Vega magnitudes of individual sources, $m_{\mathrm{F} 435 \mathrm{~W}}$ and $m_{\mathrm{F} 550 \mathrm{M}}$, following Sirianni et al. (2005). In turn, absolute magnitudes follow from our adopted distance of 2.44 Mpc and assumed intrinsic colours. In the case of sources within region A1-NW, which fell beyond the footprint of the ACS/HRC datasets, aperture photometry of radius 0.2 arcsec was performed using the ACS/WFC F606W image (Fig. 1) using GAIA.

Initially we assume uniform (foreground) interstellar extinctions of $E(B-V)=0.07$, from which we obtain absolute $F 550 M$ (or $F 606 W$ ) magnitudes. These lie in the range -6.3 to -8.4 mag for the WR sources in IC 4662. This range spans that expected by individual stars (blue supergiants) and star clusters. We discriminate between these two cases from the ACS/HRC images. The majority of WR sources are point-like, with only A2-WR2, A2-WR3 and A1-C1 spatially extended. Of course, it is possible that point sources could be compact clusters. However, given the ACS/HRC spatial resolution of $\sim 0.05 \operatorname{arcsec}(0.6 \mathrm{pc}$ at $2.44 \mathrm{Mpc}$ ), such clusters would need to be very compact to avoid detection.

The two strong He II $\lambda 4686$ emission line sources are spatially coincident with point sources A1-WR1 $\left(M_{\mathrm{F} 550 \mathrm{M}}=\right.$ $-7.6 \mathrm{mag})$ and A2-WR1 $\left(M_{\mathrm{F} 550 \mathrm{M}}=-7.9 \mathrm{mag}\right)$. Absolute visual magnitudes of individual WN stars typically lie in the range $M_{B}=-4$ to $-7 \mathrm{mag}$. The only examples of visually bright WR stars are the high mass, hydrogen-rich WN5-6ha stars for which $M_{\mathrm{B}}=-7$ to -8 mag (Crowther \& Dessart 1998). Therefore A1-WR1 and A2-WR1 are likely to be very luminous, single or binary WN5-6 stars. Their high He II $\lambda 4686$ luminosities are consistent with 1-2 luminous WN5-6 stars in each case. Such stars are located within compact, massive star clusters such as NGC 3603 in the Milky Way and R136 in the LMC, but examples are also found within more modest star forming regions, such as WR20a within Westerlund 2 in the Galaxy (Rauw et al. 2004).

A1-WR2, the other point source from aperture \#1 for which He II $\lambda 4686$ emission is detected, is rather fainter, with $M_{\mathrm{F} 550 \mathrm{M}}=$ -6.3 mag. Recalling Fig. $2 b$, this source possesses a He II line flux a factor of 2-3 times lower than A1-WR1, i.e. it is likely to be a WN5-6 star that is more typical of the SMC field population (Crowther \& Hadfield 2006), once again either single or within a binary system, unless the emission from this source were solely nebular.

The absence of high spatial resolution continuum imaging of A1-NW at more than a single continuum band prevents us discriminating between A1-WR3 and A1-WR4 for the source of He II $\lambda 4686$ emission through their colours. Both are point sources at the resolution of ACS/WFC, of which A1-WR3 is the brightest with $M_{\mathrm{F} 606 \mathrm{~W}}=-7.0 \mathrm{mag}$. In view of spectroscopy of A1-NW by Richter \& Rosa (1991), which revealed a characteristic WC4-5 spectral type, it is likely that one of A1-WR3 or A1-WR4 is a binary system comprising a WC star and OB-type companion.

For the remaining - spatially extended - sources, A2-WR2, A2-WR3 (and A1-C1) we have estimated cluster properties by first obtaining revised absolute magnitudes through comparison with the colours expected for young massive clusters at ages of 4.5 Myr. This was obtained from nebular $\mathrm{H} \beta$ line equivalent widths for STARBURST99 (Leitherer et al. 1999), based upon a Kroupa (2002) Initial Mass Function (IMF, 0.1-100 $M_{\odot}$ ) with a turnover at $0.5 M_{\odot}$, and SMC metallicity Padova evolutionary models (Fagotto et al. 1994). Such models are strictly valid when statistically large numbers of massive stars are present within individual star clusters, and break down for low cluster masses. Nevertheless, they provide a useful estimate of the likely stellar masses of individual sources should they be compact star clusters. They agree closely with the statistical approach of Parker \& Goodwin (2007) and Furness et al. (2008) in which stars are randomly sampled from the IMF.

Intrinsic $m_{\mathrm{F} 435 \mathrm{~W}}-m_{\mathrm{F} 550 \mathrm{M}}$ colours of $-0.17 \mathrm{mag}$ and -0.15 were, respectively, estimated for ages of 4.5 Myr (A2-WR2 and A2-WR3) and 6 Myr (A1-C1) from STARBURST99. Interstellar extinctions and absolute magnitudes are presented in Table 4, together with estimated cluster masses and the number of expected $\mathrm{O}$ and WR stars within individual sources. We obtain cluster mass estimates no greater than a factor of two greater than the Orion Nebula Cluster $(\mathrm{ONC})$ for which $\sim 1800 M_{\odot}$ (Hillenbrand 1997). One would expect between 3-5 O stars in such clusters, and at most one WR star in any single cluster. A2-WR2 and A2-WR3 coincide with faint He II $\lambda 4686$ emission in Fig. $2 \mathrm{~b}$ and so each likely hosts one SMC-type WN5-6 star or WC4 star.

In summary, we identify the two strong He II $\lambda 4686$ emission line sources in IC 4662 with 1-2 young, luminous WN5-6 stars (A1-WR1 and A2-WR1), and weak He II $\lambda 4686$ emission line sources with either single/binary systems (A1-WR2, A1WR3/A1-WR4) or ONC-like clusters (A2-WR2, A2-WR3). The highest mass, young, optically visible cluster IC 4662 is A1-C1, with a probable mass no greater than a factor of two higher than Orion. The few point sources in IC 4662 that are visually brighter than A1-C1 are probably yellow (AF) supergiants. IC 4662 does not appear to host any particularly young, massive, optically visible star clusters. 
Table 5. Comparison between IC $4662 \mathrm{H} \alpha$ fluxes from the literature. Inferred intensities, luminosities and Lyman continuum fluxes, follow from an adopted distance of $2.44 \mathrm{Mpc}$, and include $3 \mathrm{~cm}$ radio continuum derived ionizing flux from Johnson et al. (2003).

\begin{tabular}{lccccccc}
\hline \hline & $\begin{array}{c}\theta^{*} \\
(\operatorname{arcmin})\end{array}$ & $\begin{array}{c}F(\mathrm{H} \alpha+[\mathrm{N} \mathrm{II}]) \\
\left(\mathrm{erg} \mathrm{s}^{-1} \mathrm{~cm}^{-2}\right)\end{array}$ & $F[\mathrm{~N} \mathrm{II}] / F(\mathrm{H} \alpha)$ & $\begin{array}{c}E(B-V) \\
(\mathrm{mag})\end{array}$ & $\begin{array}{c}I(\mathrm{H} \alpha) \\
\left(\mathrm{erg} \mathrm{s}^{-1} \mathrm{~cm}^{-2}\right)\end{array}$ & $\begin{array}{c}\log L(\mathrm{H} \alpha) \\
\left(\mathrm{erg} \mathrm{s}^{-1}\right)\end{array}$ & $\begin{array}{c}Q_{0} \\
\left(10^{49} \mathrm{~s}^{-1}\right)\end{array}$ \\
\hline Kennicutt \& Kent (1983) & 2 & $7.9 \times 10^{-12}$ & 0.05 & 0.00 & $7.6 \times 10^{-12}$ & 39.73 & 400 \\
Lonsdale Persson \& Helou (1987) & 2 & $7.9 \times 10^{-12}$ & 0.05 & 0.43 & $2.1 \times 10^{-11}$ & 40.17 & 1100 \\
Helmboldt et al. (2004) & $2:$ & $1.4 \times 10^{-11}$ & 0.05 & $0.29:$ & $2.6 \times 10^{-11}$ & 40.28 & 1410 \\
Hunter \& Elmegreen (2004) & 2.2 & $1.4 \times 10^{-11}$ & 0.00 & 0.16 & $2.1 \times 10^{-11}$ & 40.17 & 1110 \\
Kennicutt et al. (2008) & 2 & $1.1 \times 10^{-11}$ & 0.08 & 0.07 & $1.2 \times 10^{-11}$ & 39.92 & 615 \\
This study & 0.2 & $5.1 \times 10^{-12}$ & 0.03 & 0.16 & $7.2 \times 10^{-12}$ & 39.71 & 380 \\
Johnson et al. (2003) & $1:$ & & & & & & 530 \\
\hline
\end{tabular}

* Approximate aperture radii.

\section{Discussion}

In this section we turn to the global properties of IC 4662, and compare it to other nearby metal-poor star forming galaxies.

\subsection{Embedded clusters in IC 4662?}

In Table 5, we compare the ACS/HRC F658N-derived number of Lyman continuum photons of IC 4662-A with previous groundbased $\mathrm{H} \alpha$ surveys of the entire galaxy, after adjustment to a common distance of $2.44 \mathrm{Mpc}$ and including corrections for the contribution of [N II] $\lambda 6548,6584$ to measured fluxes.

ACS $\mathrm{H} \alpha$ fluxes are 35-50\% lower than ground-based surveys, largely due to its much smaller field-of-view, encompassing just A1 and A2. Together, the extended sources B and C (lying to the south west and west) and D (to the south east) provide $\sim 30 \%$ of the total $\mathrm{H} \alpha$ flux of the galaxy, as noted in Sect. 2, largely explaining these differences (recall Fig. 1). A1 and A2 each possess similar $\mathrm{H} \alpha$ luminosities to the NGC 346 giant H II region in the SMC (Relaño et al. 2002).

Table 5 also includes thermal $3 \mathrm{~cm}$ continuum-derived ionizing fluxes of A1 and A2 from Johnson et al. (2003), sampling a region of IC 4662 that is similar to ACS/HRC. A comparison between these two values suggests $\sim 30 \%$ of the ionizing fluxes of A1 and A2 are deeply embedded within these regions, in agreement with Johnson et al. (2003), i.e. arguing in favour of a number of very young compact (ultradense) H II regions. However, Johnson et al. (2003) arrived at this conclusion from a comparison between their radio observations and optically derived Lyman continuum fluxes of Heydari-Malayeri et al. (1990). The latter was drawn from Lonsdale Persson \& Helou (1987), who adopted the $\mathrm{H} \alpha$ flux of Kennicutt \& Kent (1983) and a high, mean interstellar extinction of $E(B-V)=0.43 \mathrm{mag}$ for their sample of spiral and irregular galaxies. From Table 5, as a result of this assumption, their inferred number of Lyman continuum photons is a factor of 2.7 larger than Kennicutt \& Kent (1983), and a factor of two larger than Johnson et al. (2003).

In order to reconcile the optical and radio results there would need to be ionizing sources contributing to the radio-derived Lyman continuum flux of IC 4662 A1 and A2 that were undetected at $\mathrm{H} \alpha$. Young, embedded $\mathrm{H}$ II regions would be expected to be bright at both mid-IR (due to warm dust) and radio (freefree emission) wavelengths. We have therefore inspected the Spitzer/IRAC images of IC 4662 from Gilbert \& Vacca (2009) and note the presence of several IR sources that have no visual counterpart. Source N from Johnson et al. (2003) is relatively bright at $3.6 \mu \mathrm{m}$, as is a source $\sim 8$ arcsec south-west of A2HII 1, while a fainter $8 \mu \mathrm{m}$ source is seen $\sim 10$ arcsec south of A2-HII 1. Of these, solely IC4662 N represents a convincing candidate embedded ionizing source, even though it is relatively faint at $3-6 \mathrm{~cm}$. Other IRAC candidates lack radio emission, while the radio continuum sub-peak north east of A1-HII 2 has no mid-IR counterpart.

In summary, a comparison between ACS/HRC imaging of IC 4662 and previous $\mathrm{H} \alpha$ and radio surveys suggests $70 \%$ of the total ionizing flux of the galaxy originates from the giant $\mathrm{H}$ II regions A1 and A2, with a non-negligible minority of their Lyman continuum photons apparently produced by deeply embedded young massive stars, for which only IC $4662 \mathrm{~N}$ provides a realistic candidate to date, from an inspection of Spitzer/IRAC images. The majority of their ionizing budget is detected optically, consistent with the age (4.5 Myr) and stellar content (WR stars) inferred from VLT/FORS2 spectroscopy. We are unable to reconcile our results with the very high average extinction of $A_{\mathrm{V}}=$ 11-25 (9-20) mag for A1 (A2), deduced by Gilbert \& Vacca (2009) from Spitzer/IRS observations. IRAC sources mentioned above could suffer such high dust extinctions. However, since the IRS results represent averages across A1 and A2, the low average extinction of $A_{\mathrm{V}} \sim 0.5 \mathrm{mag}$ obtained for the, more luminous, optically visible $\mathrm{H}$ II regions would be expected to largely dominate the mean extinction.

The total number of WR stars in IC 4662-A1 and A2 is probably no greater than 10 if A1-WR1 and A2-WR1 indeed host $1-2$ luminous WN stars. Therefore, $N(\mathrm{WR}) / N(\mathrm{O}) \sim 0.01$, if we were to base the total number of $\mathrm{O}$ stars in $\mathrm{A} 1$ and $\mathrm{A} 2$ upon the $\mathrm{H} \alpha$ luminosity from HST/ACS and age inferred from the VLT/FORS2 H $\beta$ equivalent widths. These statistics are similar to the SMC, and are consistent with the maximum 0.02 ratio that is predicted by SMC-metallicity evolutionary models (Meynet \& Maeder 2005).

Of course, it should be bourne in mind that estimates of WR and $\mathrm{O}$ star populations suffer from significant uncertainties, namely a poorly defined WN line luminosity for SMCmetallicity stars, plus indirect $\mathrm{O}$ star numbers from nebular methods. If we were to rely solely upon the SMC metallicity WR calibration of Crowther \& Hadfield (2006), we would imply a WR population larger by one order of magnitude. Given the low statistics of WR stars in the SMC, alternative calibrations should be obtained to permit a more robust line luminosity calibration for WR stars at low metallicity. For example, IC $10-$ a SMC-metallicity star-forming galaxy within the Local Group - hosts many more Wolf-Rayet stars than the SMC (Massey \& Armandroff 1995; Crowther et al. 2003).

\subsection{IC 4662 in context}

Hunter et al. (2001) and Hunter \& Elmegreen (2004) have presented a comparison between the properties of IC 4662 and other 
Table 6. Comparison of global properties of IC 4662 with other nearby SMC-metallicity star-forming galaxies. Ionizing fluxes are taken from extinction corrected H $\alpha$ fluxes (SMC, IC4662) or radio continuum observations (IC 4662, IC 10, NGC 1569).

\begin{tabular}{|c|c|c|c|c|c|c|c|c|c|c|c|c|c|c|}
\hline Galaxy & $\begin{array}{c}d \\
(\mathrm{Mpc})\end{array}$ & Ref & $\begin{array}{c}M_{B} \\
(\mathrm{mag})\end{array}$ & $\begin{array}{c}R_{25} \\
(\mathrm{kpc})\end{array}$ & $\begin{array}{c}R_{\mathrm{D}} \\
(\mathrm{kpc})\end{array}$ & $\begin{array}{l}\log (\mathrm{O} / \mathrm{H}) \\
+12\end{array}$ & $\operatorname{Ref}$ & $\begin{array}{c}\log Q_{0} \\
\left(\mathrm{~s}^{-1}\right)\end{array}$ & Ref & $\begin{array}{l}S F R^{K 98}+ \\
\left(M_{\odot} \mathrm{yr}^{-1}\right)\end{array}$ & $\begin{array}{l}S F R^{C 08}+ \\
\left(M_{\odot} \mathrm{yr}^{-1}\right)\end{array}$ & $\begin{array}{l}S F R^{L 08} \ddagger \\
\left(M_{\odot} \mathrm{yr}^{-1}\right)\end{array}$ & $\begin{array}{c}\Sigma_{S F R}^{R_{25}} * \\
\left(M_{\odot} \mathrm{yr}^{-1} \mathrm{kpc}^{-2}\right)\end{array}$ & $\begin{array}{c}\sum_{S F R}^{R_{\mathrm{D}}} * \\
\left(M_{\odot} \mathrm{yr}^{-1} \mathrm{kpc}^{-2}\right)\end{array}$ \\
\hline SMC & 0.06 & 11 & -16.36 & 2.76 &.. & 8.13 & 1 & 51.54 & $2(\mathrm{H} \alpha)$ & 0.038 & 0.016 & 0.010 & 0.0016 &.. \\
\hline IC 10 & 0.59 & 10 & -15.45 & 0.54 & 0.34 & 8.1 & 6 & 51.61 & 7 (radio) & 0.044 & 0.019 & 0.011 & 0.049 & 0.12 \\
\hline \multirow[t]{2}{*}{ IC 4662} & 2.44 & 8 & -15.22 & 0.98 & 0.25 & 8.09 & 3 & 51.72 & 4 (radio) & 0.058 & 0.024 & 0.015 & 0.019 & 0.3 \\
\hline & & & & & & & & 51.79 & $2(\mathrm{H} \alpha)$ & 0.068 & 0.028 & 0.018 & 0.022 & 0.36 \\
\hline NGC 1569 & 3.36 & 9 & -17.98 & 1.77 & 0.38 & 8.19 & 5 & 52.80 & 2 (radio) & 0.674 & 0.287 & 0.175 & 0.07 & 1.5 \\
\hline
\end{tabular}

1. Russell \& Dopita (1990), 2. Kennicutt et al. (2008), 3. this work, 4. Johnson et al. (2003), 5. Kobulnicky \& Skillman (1997), 6. Garnett (1990),

7. Gregory et al. (1996), 8. Karachentsev et al. (2006), 9. Grocholski et al. (2008), 10. Borissova et al. (2000), 11. Hilditch et al. (2005).

† SFR prescriptions from K98 (Kennicutt 1998), C08 (Conti et al. 2008) or L08 (Leitherer 2008).

* $\Sigma_{S F R}$ for $R_{25}$ and $R_{\mathrm{D}}$ using the K98 prescription for $S F R$.

nearby dwarf irregular galaxies, while McQuinn et al. (2009) consider the recent star formation history of IC 4662 plus two other dwarf galaxies. In Table 6 we present global properties of IC 4662, based in part upon our study, with nearby galaxies exhibiting metallicities similar to that of the Small Magellanic Cloud, i.e. the SMC itself, IC 10 and NGC 1569. Of these, solely NGC 1569 is generally recognised as the nearest bonafide starburst galaxy. Absolute magnitudes, $M_{B}$ are corrected for foreground reddening and together with isophotal radii, $R_{25}$ (the $B$-band surface brightness of $25 \mathrm{mag} \operatorname{arcsec}^{-2}$ ), are drawn from de Vaucouleurs et al. (1991), while $R_{\mathrm{D}}$ was introduced by Hunter $\&$ Elmegreen (2004) as a scale length from $V$-band images ${ }^{2}$, which they considered to be a more meaningful measure of the galaxy size than $R_{25}$.

The SMC, IC 10 and IC 4662 possess similar Lyman continuum fluxes, with NGC 1569 an order of magnitude higher. As discussed above, young ionizing clusters may be deeply embedded, so radio continuum fluxes were ideally used to derive global ionizing fluxes, with the exception of the SMC, for which extinction corrected $\mathrm{H} \alpha$ fluxes were used (Kennicutt et al. 2008). The usual method of converting $\mathrm{H} \alpha$ luminosities or radio continuum fluxes into star formation rates is Kennicutt (1998). However, this approach adopts a Salpeter IMF. For an alternative Kroupa (2002) IMF, the conversion of Conti et al. (2008) should be used instead, based on Meynet et al. (1994) evolutionary models, reducing the inferred star formation rate by $\sim 60 \%$. Still lower star formation rates would be implied using contemporary metalpoor evolutionary models of Meynet \& Maeder (2005), as discussed by Leitherer (2008).

Results for all three methods are presented in Table 6, together with the star formation rate per unit area, $\Sigma_{S F R}$, as derived from the standard Kennicutt (1998) approach using both $R_{25}$ and $R_{\mathrm{D}}$. Based upon the standard Kennicutt (1998) star formation rate approach, one definition of a starburst galaxy is a star formation rate intensity in excess of $\Sigma_{S F R}^{K 98} \sim 0.1 M_{\odot} \mathrm{yr}^{-1} \mathrm{kpc}^{-2}$ (Kennicutt et al. 2005). From this definition, using the photometric radius $R_{25}$, NGC 1569 , with $\Sigma_{S F R}^{K 98}=0.07 M_{\odot} \mathrm{yr}^{-1} \mathrm{kpc}^{-2}$ would narrowly fail to be classified as starburst galaxy, followed in turn by IC 10 and IC 4662, whether using the radio flux - primarily sampling $\mathrm{A} 1$ and $\mathrm{A} 2$ - or the $\mathrm{H} \alpha$ flux of Kennicutt et al. (2008) for the latter.

Alternatively, using the definition of $R_{\mathrm{D}}$ from Hunter \& Elmegreen (2004) both NGC 1569 and IC 4662 would comfortably qualify as starbursts, with IC 10 a borderline case. Kennicutt et al. (2005) proposed that the radii of the H II regions within

\footnotetext{
${ }^{2} R_{\mathrm{D}}$ is obtained from a fit to the $V$-band surface photometry profile, $\mu=\mu_{0}+1.086 R / R_{\mathrm{D}}$ (Hunter, priv. comm.).
}

galaxies, $R_{\mathrm{HII}}$, is preferable to photometric radii when defining surface intensities - see also Martin \& Kennicutt (2001). For example, the $\mathrm{H} \alpha$ (and radio) flux of IC 4662 A1 and A2 can comfortably be accommodated within an aperture of radius $10 \operatorname{arcsec}(\sim 120 \mathrm{pc})$, from which a localised star formation intensity of $1.2 M_{\odot} \mathrm{yr}^{-1} \mathrm{kpc}^{-2}$ would be obtained. Instead, Hunter \& Elmegreen (2004) quote a $\mathrm{H}$ II radius of $R_{\mathrm{HII}}=1.14 \mathrm{kpc}$, as a result of region IC 4662-D being located far from the main body of the galaxy (recall Fig. 1).

Lee et al. (2009) use an integrated $\mathrm{H} \alpha$ equivalent width threshold of $100 \AA$ to identify bursts within dwarf galaxies. This corresponds to a stellar birthrate of $\sim 2.5$ - the current star formation rate divided by the recent average - on which basis IC 4662 represents a borderline starburst, with an equivalent width of $\sim 101 \pm 10 \AA$. Within the local volume $(11 \mathrm{Mpc})$ only a handful of dwarf galaxies fainter than $M_{B} \sim-17$ mag would be characterised as starbursts on this basis, including NGC 1569 and other well known cases NGC 1705, NGC 2366, NGC 3125 and NGC 5253.

\section{Conclusions}

We have carried out a ground-based VLT/FORS2 imaging and spectroscopic survey of the massive stellar content of giant $\mathrm{H}$ II regions $\mathrm{A} 1$ and $\mathrm{A} 2$ within the nearby star forming galaxy IC 4662, supplemented by archival HST/ACS imaging.

From a comparison between radio continuum observations of IC 4662 with HST/ACS H $\alpha$ imaging, we find that a minority of the ionizing fluxes of A1 and A2 likely result from deeply embedded clusters, in agreement with conclusions of Johnson et al. (2003). A 1 and A2 each possess similar $\mathrm{H} \alpha$ line luminosities to NGC 346 in the SMC.

We identify several sources hosting Wolf-Rayet stars (or nebular He II $\lambda 4686$ emission). There is one bright and one faint source within each of $\mathrm{A} 1$ and $\mathrm{A} 2$ plus one faint region $\sim 8$ arcsec north west of A1 that we label A1-NW. Spectroscopy reveals that mid-type WN stars are the dominate subtypes within A1 and A2, in addition to one WC star in each of $\mathrm{A} 2$ and $\mathrm{A} 1-\mathrm{NW}$ together with nebular He II $\lambda 4686$ emission in two instances. ACS/HRC imaging permits individual sources responsible for emission line features to be identified, which we conclude are a mixture of luminous WN stars (or WR binaries) and 5 Myr old clusters, with masses up to two times larger than the Orion Nebula Cluster. In contrast with other nearby dwarf irregular galaxies exhibiting starburst activity, IC 4662 appears to lack young, optically bright very massive clusters. For example, the brightest young cluster of IC 4662 is A1-WR2 for which $M_{\mathrm{F} 550 \mathrm{M}}=-9$ mag, versus 
$M_{\mathrm{F} 555 \mathrm{~W}}=-11.9 \mathrm{mag}$ for the $5 \mathrm{Myr}$ old cluster NGC $1569 \mathrm{~A} 2$ (Maoz et al. 2001; Sirianni et al. 2005).

Finally, we compare the global properties of IC 4662 with other nearby SMC-metallicity star forming galaxies. Based upon photometric radius $R_{25}$, IC 4662 is not a starburst galaxy, even though its global star formation intensity is an order of magnitude higher than that of the SMC. NGC 1569 and IC 10 possess star formation intensities a factor of $\sim 3$ times higher than IC 4662 , the former generally recognised as the nearest bonafide starburst galaxy. If we were to follow the approach of Hunter $\&$ Elmegreen (2004), by defining an alternative scale length, $R_{\mathrm{D}}$, measured from $V$-band images to define the size of the galaxy, both NGC 1569 and IC 4662 would readily qualify as starbursts - see also Lee et al. (2009).

Wolf-Rayet stars within low metallicity irregular galaxies such as IC 4662 are leading candidates for type Ic supernovaGRBs (Fruchter et al. 2006; Hammer et al. 2006; Modjaz et al. 2008). Establishing the spatial location of WR stars within such environments would permit the progenitors of such explosions, in the case of a core-collapse supernova fortuitously occuring in the near future within IC 4662.

Acknowledgements. Thanks to Hans Schild for co-observing IC 4662, Janice Lee for permitting use of unpublished CTIO $\mathrm{H} \alpha$ images of IC 4662, Deidre Hunter for details of her $\mathrm{H} \alpha$ observations, and to Bill Vacca for useful discussions. J.L.B. acknowledges financial support from STFC. This research has made extensive use of the NASA/IPAC Extragalactic Database (NED) which is operated by the Jet Propulsion Laboratory, California Institute of Technology, under contract with the National Aeronautics and Space Administration.

\section{References}

Borissova, J., Georgiev, L., Rosado, M., et al. 2000, A\&A, 363, 130

Buckalew, B. A., \& Kobulnicky, H. A. 2006, AJ, 132, 1061

Conti, P. S., Crowther, P. A., \& Leitherer, C. 2008, From Luminous Hot Stars to Starburst Galaxies, Cambridge Astrophysics Series (Cambridge: Cambridge University Press), 45

Crowther, P. A., \& Dessart, L. 1998, MNRAS, 296, 622

Crowther, P. A., \& Hadfield, L. J. 2006, A\&A, 449, 711

Crowther, P. A., Drissen, L., Abbott, J. B., Royer, P., \& Smartt, S. J. 2003, A\&A, 404, 483

De Robertis, M. M., Dufour, R. J., \& Hunt, R. W. 1987, JRASC, 81, 195

de Vaucouleurs, G., de Vaucouleurs, A., Corwin, Jr., H. G., et al. 1991, Third Reference Catalogue of Bright Galaxies (Berlin: Springer-Verlag)

Fagotto, F., Bressan, A., Bertelli, G., \& Chiosi, C. 1994, A\&AS, 105, 29

Fruchter, A. S., Levan, A. J., Strolger, L., et al. 2006, Nature, 441, 463

Furness, J. P., Crowther, P. A., Conti, P. S., Rho, J., \& Goodwin, S. P. 2009, in prep.

Garnett, D. R. 1990, ApJ, 363, 142

Gilbert, A. M., \& Vacca, W. D. 2009, in Young Massive Star Clusters: Initial Conditions and Environments, ed. R. Perez, R. de Grijs, \& R. M. González Delgado, Astrophysics and Space Science, in press

[arXiv:0806.2302]
Gregory, P. C., Scott, W. K., Douglas, K., \& Condon, J. J. 1996, ApJS, 103, 427 Grocholski, A. J., Aloisi, A., van der Marel, R. P., et al. 2008, ApJ, 686, L79 Hadfield, L. J., \& Crowther, P. A. 2006, MNRAS, 368, 1822

Hammer, F., Flores, H., Schaerer, D., et al. 2006, A\&A, 454, 103

Helmboldt, J. F., Walterbos, R. A. M., Bothun, G. D., O’Neil, K., \& de Blok, W. J. G. 2004, ApJ, 613, 914

Heydari-Malayeri, M., Melnick, J., \& Martin, J.-M. 1990, A\&A, 234, 99

Hidalgo-Gámez, A. M., Masegosa, J., \& Olofsson, K. 2001, A\&A, 369, 797

Hilditch, R. W., Howarth, I. D., \& Harries, T. J. 2005, MNRAS, 357, 304

Hillenbrand, L. A. 1997, AJ, 113, 1733

Hummer, D. G., \& Storey, P. J. 1987, MNRAS, 224, 801

Hunter, D. A., \& Elmegreen, B. G. 2004, AJ, 128, 2170

Hunter, D. A., Kaufman, M., Hollenbach, D. J., et al. 2001, ApJ, 553, 121

Johnson, K. E., Indebetouw, R., \& Pisano, D. J. 2003, AJ, 126, 101

Karachentsev, I. D., Dolphin, A., Tully, R. B., et al. 2006, AJ, 131, 1361

Kennicutt, Jr., R. C. 1998, ARA\&A, 36, 189

Kennicutt, Jr. R. C., \& Kent, S. M. 1983, AJ, 88, 1094

Kennicutt, Jr., R. C., Lee, J. C., Funes, J. G., Sakai, S., \& Akiyama, S. 2005, in Starbursts: From 30 Doradus to Lyman Break Galaxies, ed. R. de Grijs, \& R. M. González Delgado, ASSL, 329, 187

Kennicutt, Jr., R. C., Lee, J. C., Funes, J. G. S. J., Sakai, S., \& Akiyama, S. 2008, ApJS, 178, 247

Kobulnicky, H. A., \& Skillman, E. D. 1997, ApJ, 489, 636

Kroupa, P. 2002, Science, 295, 82

Lee, J. C., Kennicutt, Jr., R. C., Funes, J. G. S. J., Sakai, S., \& Akiyama, S. 2009, ApJ, 692, 1305

Leitherer, C. 2008, in Low-Metallicity Star Formation, ed. L. Hunt, S. Madden, \& R. Schneider, IAU Symp., 255, 305

Leitherer, C., Schaerer, D., Goldader, J. D., et al. 1999, ApJS, 123, 3

Lonsdale Persson, C. J., \& Helou, G. 1987, ApJ, 314, 513

Maoz, D., Ho, L. C., \& Sternberg, A. 2001, ApJ, 554, L139

Martin, C. L., \& Kennicutt, Jr., R. C. 2001, ApJ, 555, 301

Massey, P., \& Armandroff, T. E. 1995, AJ, 109, 2470

McQuinn, K. B. W., Skillman, E. D., Cannon, J. M., et al. 2009, ApJ, 695, 561

Meynet, G., \& Maeder, A. 2005, A\&A, 429, 581

Meynet, G., Maeder, A., Schaller, G., Schaerer, D., \& Charbonnel, C. 1994, A\&AS, 103, 97

Modjaz, M., Kewley, L., Kirshner, R. P., et al. 2008, AJ, 135, 1136

Osterbrock, D. E., \& Ferland, G. 2006, Astrophysics of Gaseous Nebulae and Active Galactic Nuclei, 2nd edn. (Maple Vail: University Science Books)

Parker, R. J., \& Goodwin, S. P. 2007, MNRAS, 380, 1271

Prescott, M. K. M., Kennicutt, Jr., R. C., Bendo, G. J., et al. 2007, ApJ, 668, 182 Rauw, G., De Becker, M., Nazé, Y., et al. 2004, A\&A, 420, L9

Relaño, M., Peimbert, M., \& Beckman, J. E. 2002, in Rev. Mex. Astron. Astrofis. Conf. Ser., ed. W. J. Henney, J. Franco, \& M. Martos, 12, 263

Richter, O.-G., \& Rosa, M. R. 1991, in Wolf-Rayet Stars and Interrelations with Other Massive Stars in Galaxies, ed. K. A. van der Hucht, \& B. Hidayat, IAU Symp., 143, 653

Rosa, M., \& Dodorico, S. 1986, in Luminous Stars and Associations in Galaxies, ed. C. W. H. de Loore, A. J. Willis, \& P. Laskarides, IAU Symp., 116, 355

Russell, S. C., \& Dopita, M. A. 1990, ApJS, 74, 93

Schaerer, D., \& Vacca, W. D. 1998, ApJ, 497, 618

Schaerer, D., Contini, T., \& Pindao, M. 1999, A\&AS, 136, 35

Schlegel, D. J., Finkbeiner, D. P., \& Davis, M. 1998, ApJ, 500, 525

Seaton, M. J. 1979, MNRAS, 187, 73P

Sirianni, M., Jee, M. J., Benítez, N., et al. 2005, PASP, 117, 1049

Tody, D. 1986, in Instrumentation in Astronomy VI, ed. D. L. Crawford, SPIE, 627,733

Vacca, W. D. 1994, ApJ, 421, 140 